\title{
Orbital Stability Zones about Asteroids
}

\author{
Douglas P. Hamilton and Joseph A. Burns \\ Cornell University, Ithaca, New York, 14853-6801
}

Received October 5, 1990; revised March 1, 1991

\begin{abstract}
We have numerically investigated a three-body problem consisting of the Sun, an asteroid, and an infinitesimal particle initially placed about the asteroid. We assume that the asteroid has the following properties: a circular heliocentric orbit at $R=2.55 \mathrm{AU}$, an asteroid/Sun mass ratio of $\mu=5 \times 10^{-12}$, and a spherical shape with radius $R_{\mathrm{A}}=100 \mathrm{~km}$; these values are close to those of the minor planet 29 Amphitrite. In order to describe the zone in which circum-asteroidal debris could be stably trapped, we pay particular attention to the orbits of particles that are on the verge of escape. We consider particles to be stable or trapped if they remain in the asteroid's vicinity for at least 5 asteroid orbits about the Sun, or about 20 years. Applying this criterion to particles started on circular orbits around the asteroid, we find that, as the starting distance from the asteroid is varied, a fairly abrupt transition between trapped and untrapped objects occurs. We define the distance where the transition occurs to be the critical distance.
\end{abstract}

Our orbital plots for Amphitrite can be scaled for application to other asteroids using the same functional dependence as the Hill radius which is $r_{H}=(\mu / 3)^{1 / 3} R$; for Amphitrite $r_{H} \sim 450 R_{A}$. We find empirically that initially circular prograde orbits remain bound out to a critical distance of about $\left(r_{H}\right) / 2=225 R_{A}$, while initially circular retrograde orbits remain bound out to nearly twice that distance. Particle orbits that start out circular and are inclined with respect to the asteroid's orbital plane have critical distances between these two extremes. Note that our choice of initially circular orbits is arbitrary; different initial conditions would generally lead to different critical distances.

This study explores the three-dimensional aspects of stability more thoroughly than previous studies. To first order, particles that are on stable orbits reside within a region that is approximately spherical for angles $|\theta|<35^{\circ}$ ( $\theta$ is the latitude in a spherical coordinate system) such that $|z|<285 R_{\mathrm{A}}$, but is fairly flat and parallel to the $x-y$ plane at $z= \pm 285 R_{\mathrm{A}}$ over the poles. The radius of the spherical surface is roughly $480 R_{\mathrm{A}}$. These distances would be reduced if the model included the asteroid's orbital eccentricity or other perturbations such as those from Jupiter. This result does not address whether any mechanisms exist to populate such orbits. 1991 Academic Press, Inc.

\section{INTRODUCTION}

Spacecraft have now visited all classes of solar system targets, excepting asteroids. This neglect of the planetary exploration program should be remedied when the Galileo spacecraft, launched in October 1989, encounters the asteroid 951 Gaspra on October 29, 1991. It is expected that the asteroid's surroundings will be almost devoid of material and therefore benign since, in the analogous case of the satellites of the giant planets, significant debris has never been detected. The analogy is imperfect, however, and so the possibility that interplanetary debris may be enhanced in the gravitational well of the asteroid must be considered. If this is the case, the danger of collision with orbiting debris may increase as the asteroid is approached.

Since the character of the circum-asteroidal environment cannot be well characterized from ground-based observations (Gradie et al. 1985, Terrile and Smith 1985), some have argued that the distance of closest approach of the first exploratory space mission should be chosen very conservatively. Countering this cautious point of view is the fact that several instruments aboard the Galileo spacecraft, notably the visual imaging and especially the infrared imaging systems, would benefit by a close flyby. Hence, in order to properly weigh the scientific gain of a close asteroidal approach against the increased risk of a catastrophic collision with unseen circum-asteroidal debris, we have sought to determine those regions near an asteroid where material may orbit stably. Although not discussed in this article, the question of how circum-asteroidal debris might be generated must be addressed before one can determine the likelihood that material might reside near an asteroid.

Since the problem of $N$ gravitationally attracting bodies is well known to be analytically unsolvable for $N>2$, numerical methods must be employed to obtain quantitative estimates of the motion of a test particle in the vicinity of an asteroid that itself circles the Sun. The three-body problem has been numerically integrated many times previously (consult Szebehely (1967) for historical references while for more recent work see Zhang and Innanen (1988), Murison (1989b), Chauvineau and Mignard (1990a,b)) but the space of possible parameters is so large that the threebody problem's complete solution is, fundamentally, not understood. Fortunately the problem that we wish to solve is more restricted, although still analytically intractable. 
We treat the case of hierarchical masses since the asteroid's mass is insignificant relative to the solar mass, yet is very large in comparison to particles likely to be orbiting it. Hierarchical masses provide a limiting case of both Hill's problem and the restricted three-body problem (Hénon and Petit 1986). We further narrow the space of parameters by giving the asteroid a circular orbit around the Sun, by choosing to study only those orbits that are weakly bound to the asteroid, and by starting test particles out on initially circular orbits. The second choice is made in order to explore the transition region between bound and unbound orbits and hence to delineate the zone in which the material could be stably trapped.

In the numerical examples to follow, we model the asteroid 29 Amphitrite, a previously planned target of Galileo (see also Zhang and Innanen 1988), as having a circular orbit of radius $R=2.55 \mathrm{AU}$, and an asteroid/Sun mass ratio $\mu=5.0 \times 10^{-12}$. For an assumed asteroid radius of $100 \mathrm{~km}$, the chosen $\mu$ corresponds to a reasonable density of $2.38 \mathrm{~g} / \mathrm{cm}^{3}$. Our investigation confirms and extends the study of Zhang and Innanen (1988) by using heuristic models to understand the nature of the observed orbits, by considering motion out of the orbital plane, by illustrating the shape of the volume filled by particles on stable orbits, by showing how results can be scaled to other asteroids, and by placing the problem in the context of modern ideas on chaos (Chauvineau and Mignard 1990a,b, Murison 1989b).

\section{EQUATION OF MOTION}

We use two noninertial coordinate systems (Fig. 1), each with its origin on the asteroid which itself orbits the Sun: nonrotating coordinates that keep their axes fixed with respect to the distant stars, and rotating coordinates that maintain their axes fixed relative to the Sun. In each, the asteroid's orbit lies in the $x-y$ plane. Because the orbits we consider are only weakly bound to the asteroid, solar perturbation forces are relatively large and, accordingly, most paths are more easily understood when viewed in a reference frame rotating with the asteroid's mean motion $\Omega \hat{\mathbf{z}}$ around the Sun $\left(x_{\text {rot }}, y_{\text {rot }}, z\right.$ in Fig. 1). The mean motion is a vector that points normal to the orbit ( $\hat{\mathbf{z}}$ is the unit vector in the $z$ direction) and has magnitude

$$
\mathbf{\Omega}=\sqrt{\frac{\mathrm{GM}}{R^{3}}},
$$

where $G$ is the gravitational constant, $R$ is the Sun-asteroid distance, and $M$ is the mass of the Sun. The acceleration $\ddot{\mathbf{r}}$ of a particle orbiting the asteroid is then approximately given by Hill's equation (Szebehely, 1967),

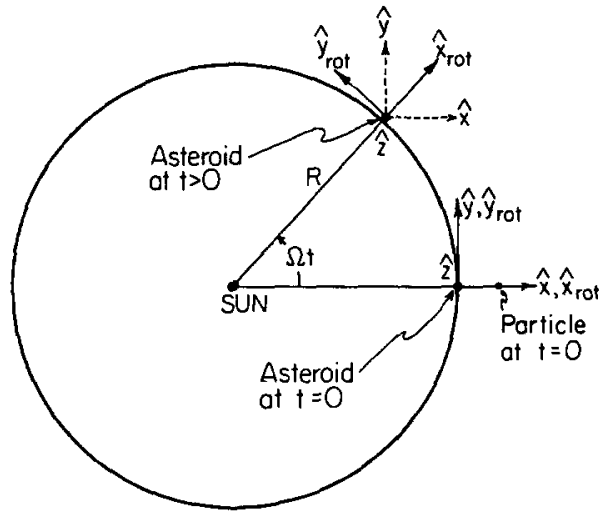

FIG. 1. Two noninertial coordinate systems are shown as they follow the asteroid on its circular orbit of radius $R$ about the Sun. The $x y z$ system stays fixed in its angular orientation while the $(x y z)_{\text {rot }}$ system rotates uniformly so that the Sun always is at $x_{\text {rot }}=-R$. In the nonrotating system the Sun is initially at $x=-R$ and it moves with angular velocity $\Omega$ around the asteroid in the plane $z=0$. In most integrations the particle starts along the Sun-asteroid line at $(x=d, y=0, z=0)$ with a velocity in the nonrotating frame that would put it on a circular orbit if the Sun were not present.

$$
\ddot{\mathbf{r}}=-\frac{G M_{\mathrm{A}}}{r^{2}} \hat{\mathbf{r}}+\Omega^{2}\left(3 x_{\text {rot }} \hat{\mathbf{x}}_{\text {rot }}-z \hat{\mathbf{z}}\right)-2 \mathbf{\Omega} \times \mathbf{v}_{\text {rot }}
$$

where $\mathbf{r}$ is the vector pointing from the asteroid to the particle, $\hat{\mathbf{r}}$ is the corresponding unit vector, $\mathbf{v}_{\text {rot }}$ is the particle's velocity measured in the rotating frame, and $M_{\mathrm{A}}$ is the mass of the asteroid. The terms on the right side of Eq. (2) are due to the asteroid's direct gravity, the combination of solar tidal and centripetal effects, and the Coriolis effect, respectively. Henceforth, the full second term will be referred to as the "tidal" term. In the derivation of Eq. (2), we have neglected quantities that are second order in $r / R$. These terms, if included, would break the symmetry of the tidal term around the $y_{\text {rot }}-z$ plane. We have observed consequences of this broken symmetry in a few escape orbits, but do not judge it to be significant in estimating the trapped region or in describing most orbits.

In order for the reader to gain insight into the trajectories to be shown later, we now discuss some of the properties of the accelerations in Eq. (2). In these descriptions we will call an orbit prograde if the particle's angular velocity around the asteroid is in the same sense as the asteroid's angular velocity around the Sun; for a retrograde orbit, the particle's angular velocity is in the opposite sense. Figure 2 is a sketch showing how the direction and magnitude of the various accelerations change along a hypothetical orbit that is coplanar and oval-shaped in the rotating frame. Notice that the accelerations all act in different directions: the direct term always points toward the asteroid, the tidal term invariably aligns parallel or antiparallel to the solar direction, and the Coriolis term is 
DIRECT ACCELERATION
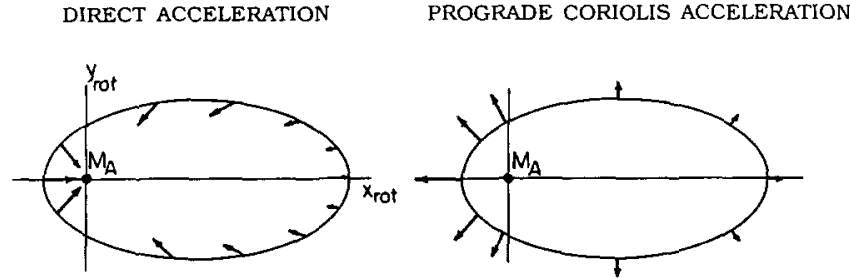

TIDAL ACCELERATION

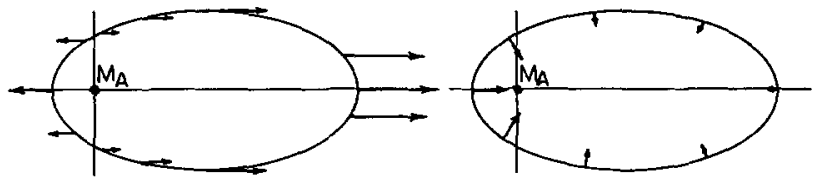

FIG. 2. Sketches of the accelerations (magnitudes and directions) that are experienced by a particle at various places along a coplanar oval orbit whose long axis is aligned with the solar direction; the asteroid is at the origin. The direct acceleration is caused by the asteroid's gravitational attraction of the particle. The "tidal" acceleration is due to the local imbalance between the Sun's attraction and that needed to cause the asteroid's circular path [see Eq. (2)]. The sign of the Coriolis acceleration depends on whether the particle moves in the same (prograde) or opposite (retrograde) angular sense as the asteroid in its orbit about the Sun.

always perpendicular to the orbit. Furthermore, the direct acceleration is inward and thus acts to bind particles to the asteroid, while the tidal acceleration, which has a component that points outward, acts to expel them from the system. The Coriolis acceleration points outward for prograde orbits but inward for retrograde ones; thus it tends to stabilize the latter but disrupt the former.

Finally note that along a given orbit the tidal acceleration increases with growing separation distance, while all other accelerations decrease. Comparing the directions of the accelerations in the prograde and retrograde cases, we can already see that retrograde orbits should be stable out to greater distances than prograde ones since in the former situation the Coriolis acceleration is inward while in the latter it is outward. Numerical experiments support this statement as does nature's laboratory: the outermost moons of Jupiter and Saturn are on retrograde orbits.

In order to quantify the radial dependences of the accelerations, they are plotted in Fig. 3 as functions of distance from the asteroid for the special case of a circular coplanar orbit. Recall that the asteroid's mean density is assumed to be $2.38 \mathrm{~g} / \mathrm{cm}^{3}$ and its semimajor axis is assumed to be $2.55 \mathrm{AU}$. To adjust the axes of this and all of the following plots to your favorite asteroid, simply multiply distances measured in asteroid radii by the factor $(R / 2.55)$ $(\rho / 2.38)^{1 / 3}$, where $\rho$ is the asteroid's density in grams per cubic centimeter and $R$ is its semimajor axis in AU. The justification for this scaling will be presented in a later section; we also note here that differences in asteroid orbital eccentricities are not, and cannot be, accommodated.
All of the curves plotted in Fig. 3 are normalized by the local direct acceleration of the asteroid's gravity. Since the strength of the tidal acceleration depends on azimuthal position (see Fig. 2), it varies along even a circular orbit and thus here we plot its maximum value. The total accelerations for prograde [curve $\mathrm{P}$ ] and retrograde [curve $\mathrm{R}$ ] orbits as plotted in Fig. 3 were obtained by taking the various terms and simply adding them; even though this addition ignores the vector character of these accelerations, we believe that it is instructive.

In the limit of small separations (i.e., on the left side of Fig. 3), the perturbation accelerations [curves $C(P), C(R)$, and $\mathrm{T}]$ tend to zero, and thus both prograde and retrograde orbits approach the two-body solutions: circles and ellipses about the asteroid. Accordingly, the curves of Fig. 3 are most applicable in this inner region, since only there do circular orbits actually exist. Nevertheless, the curves provide useful guides for estimating magnitudes in more complicated situations. Of course, care must be exercised in their application, especially when estimating the magnitude of the Coriolis acceleration which, due to its velocity dependence, will vary substantially with the actual path taken.

Both the restricted three-body problem and Hill's problem admit an integral of the motion that can be derived by integrating, over time, the scalar product of Eq. (2) with the velocity $\mathbf{v}_{\text {rot }}$. Chauvineau and Mignard's expression (1990a) for this "Jacobi" integral can be generalized to three dimensions as

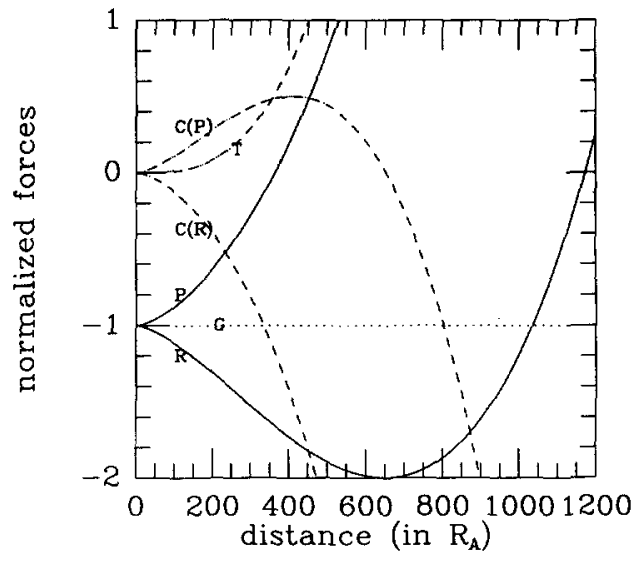

FIG. 3. The various accelerations acting on a particle as it moves along a circular coplanar orbit about the asteroid are plotted versus separation from the asteroid; all accelerations are normalized to $G$, the local gravitational attraction of the asteroid, which decreases as the inverse square of the separation. The dashed curves show the various perturbations, which are all zero for orbits atop the asteroid (i.e., at zero separation); $T$ is the maximum "tidal" term, C(P) is the prograde Coriolis acceleration and $C(R)$ is the retrograde Coriolis acceleration. $P$ and $\mathbf{R}$ are the total perturbations that act on prograde and retrograde particles, respectively, ignoring the vector nature of the actual forces. 


$$
\left(\mathrm{v}_{\mathrm{rot}}\right)^{2}-\Omega^{2}\left(3 x^{2}-z^{2}\right)-\left(2 G M_{\mathrm{A}} / r\right)=-C .
$$

where $C$ is the Jacobi constant and $r=\left(x^{2}+y^{2}\right.$ $\left.+z^{2}\right)^{1 / 2}$ is the distance of the particle to the asteroid. Our Jacobi constant is conserved in a rotating frame centered on the asteroid and is related to the more usually defined Jacobi constant (see Szebehely 1967) which is conserved in a rotating frame with its origin at the systems center of mass. Subsequently we will give the quantity $-C / 2$ the name "energy" to distinguish it from the heliocentric energy (energy of a body orbiting the Sun) and the twobody energy (energy of a body orbiting the asteroid if the Sun were not present). Since a particle's velocity must always remain real, and since $C$ is fixed uniquely by initial conditions (position and speed), Eq. (3) restricts the motion of any particle to lie within those regions of space where the following inequality is satisfied:

$$
\Omega^{2}\left(3 x^{2}-z^{2}\right)+\left(2 G M_{\mathrm{A}} / r\right)>C .
$$

The lines along which the velocity is zero (i.e., those places where the left-hand side of Eq. (4) equals C) are called zero-velocity or Hill curves. An escape criterion that can been invoked is that whenever, for given initial conditions, a particle lies within a zero-velocity surface that is closed about the asteroid, the particle cannot escape that region. Of course the converse does not hold: there is no guarantee that, just because the Hill curve is open, the particle will necessarily escape in a finite time. The distance to the positions along the $x_{\text {rot }}$ axis at which the zero-velocity surface first opens can be computed to be $r_{\mathrm{H}}=(\mu / 3)^{1 / 3} R$ for Hill's problem (Danby 1988). These points are two of the three co-linear Lagrange points (the other is on the far side of the Sun) and their distance from the asteroid defines the radius of the Hill sphere. The colinear Lagrange points are unstable equilibrium points; a particle placed with zero velocity in one of these positions will remain there forever, but particles starting arbitrarily close will depart the neighborhood.

\section{GENERAL REMARKS ON THE SOLUTION}

\section{Integrations}

Our numerical integrations call upon an efficient integrator that utilizes both the Bulirsch-Stoer and Runge-Kutta methods (Press et al. 1987). The routine takes advantage of the speed of the Bulirsch-Stoer technique, falling back on the Runge-Kutta scheme during close approaches between the two bodies (cf. Murison 1989a).

In our integrations the particle was generally started along the Sun-asteroid line, on the far side of the minor planet (Fig. 1). It was usually given a velocity that would place it on a circular orbit around the asteroid if perturbations from the Sun were absent. In many simulations the plane of the particle's orbit was given an initial inclination $i$ with respect to the plane of the asteroid's orbit. The inclination is positive to the heliocentric north, and reaches $180^{\circ}$ for a purely retrograde orbit. With these initial conditions, the ones used most frequently, the only degrees of freedom are the initial separation distance and the initial inclination. We also explored other initial conditions for the particle to assess the generality of our results. In these explorations, we altered one or more of the following: the starting longitude, the direction of initial velocity, and the initial speed. We also ran a large number of tests with an asteroid whose orbit around the Sun has a moderate eccentricity. The effects of orbital eccentricity, as well as the forces from Jupiter and radiation pressure, will be discussed in a later paper.

\section{Nature of Orbits}

Since the relative strengths of the various perturbations change with separation (Fig. 3), orbits may have quite different characteristics depending on their distances from the asteroid (Chauvineau and Mignard 1990a). Within a few asteroidal radii, orbits are simple Keplerian ellipses since the asteroid's gravity dominates all perturbations (see Fig. 3 and the earlier discussion). Farther out, perturbations become large enough to induce orbital planes and pericenters to precess noticeably, although the orbits retain their basic Keplerian nature. As the distance is increased still further we come to a region in which quasiperiodic stable orbits are intermingled with chaotic paths. An orbit is quasiperiodic if it contains only a finite number of incommensurate frequencies. In many of our experiments the period corresponding to the particle's dominant frequency is seen to be commensurate with the asteroid's orbital period; such a commensurate "locking" between the forcing frequency and the natural response of a system is a common feature of nonlinear systems.

This quasiperiodic/chaotic zone gradually gives way to the realm of escape orbits which we define as those trajectories that depart the vicinity of the asteroid, but the division between these regions is not clearly defined; in fact, in the circular restricted three-body problem the boundary between these regions is self-similar in a fractallike manner (Murison 1989b). In an area where escape orbits predominate, isolated "islands" of stable quasiperiodic orbits can occur (Chauvineau and Mignard, 1990a). And, likewise, in regions where mostly quasiperiodic orbits exist, a few escape orbits can be found. Although the regions are not entirely disconnected, we observe that beyond a certain "stability boundary," the number of stable orbits drops very sharply. Our ultimate goal is to understand the size and shape of this boundary that sepa- 
rates orbits bound to the asteroid from those that escape its influence. Since chaotic orbits are prevalent in the transition zone, our results for the size of the stability zone are probably conservative: longer integrations would have shown additional escapes (Wisdom, 1982). But, to a first approximation, we can determine the locus of points forming the stability boundary by looking at the outermost regions where the majority of orbits are stable. Chaos necessarily permeates these outer regions, since a particle's fate certainly depends sensitively on initial conditions (Murison 1989b).

Since the results of Chauvineau and Mignard (1990a), which follow on the pioneering study of Hénon (1970), are so relevant to our findings, they will be summarized here. These authors use the surface-of-section technique to study the stability of motions in Hill's problem. They find that, for prograde orbits that have a Jacobi constant much greater than the critical value at which the Hill curves no longer enclose the asteroid, the motions are regular: trajectories are nearly periodic, and stable. In nondimensional units, $G=1, \Omega=1$, and $r_{\mathrm{H}}=1$ (these choices set $M_{\mathrm{A}}=3$ ), and the critical Jacobi constant occurs at $C=$ 9. At values somewhat above 9 (from 9.2 to 9.3604 to be precise), the topological structure of the mapping is such that new periodic orbits are introduced as $C$ is lowered; more and more of these periodic islands appear as 9.2 is approached and the regularity of the mapping is lost. At 9.2 and below, chaotic trajectories appear in parts of the mapping. These ergodic regions tend to fill up more and more of the phase space until, with $C$ near 9, little of the surface of section is populated with periodic islands; instead virtually all is a sea of chaos. Note that up to this point, since all the zero-velocity curves corresponding to $C>9$ encircle the asteroid, the motions are bounded with the particles remaining about the asteroid, albeit moving along chaotic paths. However, once the Jacobi constant falls below 9, suddenly the ergodic region becomes connected with external parts of the phase space. That is, however, not to say that all particles will necessarily escape in a finite time, merely that it is energetically possible for particles with $C<9$ to find their way through the ergodic region and escape. Some regular direct orbits do exist for $8.88<C<9.00$, although they cover little of the available phase space. For retrograde orbits Chauvineau and Mignard (1990a) find quite different results. With $C \gg 9$ the mapping is usually regular and, as in the prograde case, chaos appears when $C$ is a bit larger than 9 . The striking difference is that many regular retrograde orbits are seen to persist for values of $C$ well below the critical value, unlike the prograde situation. For completeness, we note that there are also a small number of pathological orbits that oscillate between the direct and retrograde states.

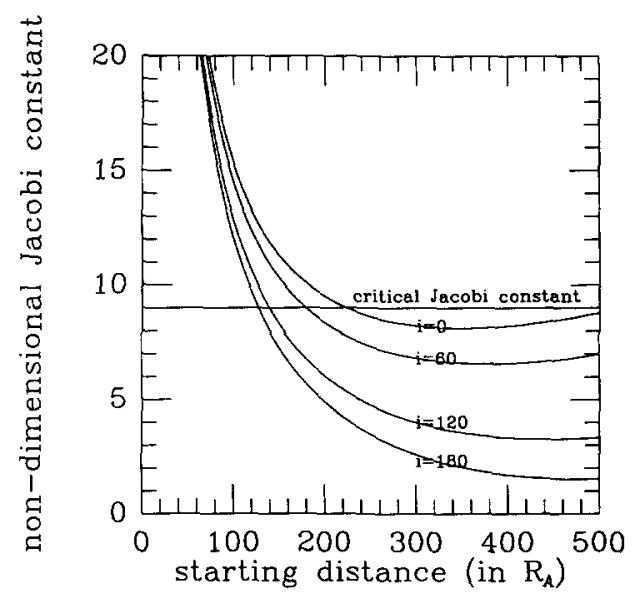

FIG. 4. The Jacobi constant, in nondimenstional units $(G=1$, $\Omega=1, r_{\mathrm{H}}=1$ ), is plotted for the family of orbits studied in this paper. These orbits are initially circular, are started from the positive $x$ axis, and are inclined by an angle $i$ with respect to the $x-y$ plane. The critical Jacobi constant $(C=9)$ is also plotted. If the Jacobi constant of a particular orbit lies above the critical line, that particle is bound to the asteroid for all time. If, however, it lies below the critical line, the particle is energetically able to escape, although it is not required to do so.

To help the reader connect the results of Chauvineau and Mignard (1990a) to the trajectories that we will be plotting later, we now show that a one-to-one correspondence exists between our usual initial conditions and the Jacobi constant. Recall that we start a particle at $(d, 0,0)$, with a velocity that is inclined at an angle $i$ from the $x-y$ plane and whose speed in the nonrotating frame is $\left(G M_{\mathrm{A}} / d\right)^{1 / 2}$. From Eq. (3) the Jacobi constant for this initial condition is

$$
\begin{array}{r}
C=-\left[\left(G M_{\mathrm{A}} / d\right)^{1 / 2} \cos (i)-\Omega d\right]^{2}-\left(G M_{\mathrm{A}} / d\right) \sin ^{2}(i)+3 d^{2} \Omega^{2} \\
+2 G M_{\mathrm{A}} / d
\end{array}
$$

Figure 4 is a plot of $C$ versus the starting distance $d$ for various inclinations $i$; the plotted Jacobi constant is given in the nondimensional units used by Chauvineau and Mignard (1990a).

\section{Scaling to Other Asteroids}

Even though most of our simulations considered a specific case $\left(\mu=5 \times 10^{-12}\right.$ and $\left.R=2.55 \mathrm{AU}\right)$, we can apply our results to other asteroids with different semimajor axes and mass ratios. Consider a system of $N$ gravitationally interacting bodies viewed from an inertial frame. All forces in the system are gravitational, so the strength of each interaction varies as the inverse square of distance. In particular, if all distances are multiplied by a factor $\alpha$, 
the forces retain their directions and are reduced by $\alpha^{2}$. One can then rescale time so that the resulting system of differential equations is identical to the original set: therefore, as long as the initial velocities are also appropriately modified, identical orbital paths will result. So, for example, if the asteroid's distance from the Sun is doubled, particle orbits around the asteroid will have the same shape as in the original case if starting distances from the asteroid are doubled and velocities are reduced by a factor of $2^{1 / 2}$. Thus, the orbits scale with the asteroid's semimajor axis $R$.

Employing similar ideas to a change in the asteroid's mass, we find that the orbit scales with $\mu^{1 / 3}$ for the case of the three-body Hill problem with the asteroid-particle distance much less than the distance to the Sun. This approximation is well satisfied for the motion of bound particles. When combined, these two results imply the powerful assertion that for each orbit existing around one asteroid, a corresponding orbit, differing only in absolute size, exists around a second asteroid provided that the two asteroids have the same orbital eccentricity. The ratio of the sizes of the two orbits is equal to the ratio of the radii of their respective Hill spheres: $(\mu / 3)^{1 / 3} R$. If the sizes are measured in asteroid radii, as in our plots, they scale as $\rho^{1 / 3} R$. In particular the orbital stability zone, which is the union of all stable orbits, scales as this ratio. For Amphitrite, the Hill sphere has a radius of $\sim 450 R_{\mathrm{A}}$, while for Gaspra it is $\sim 390 R_{\mathrm{A}}$.

At any rate, it is clear that Hill sphere scaling differs from $\mu^{2 / 5} R$, the size of the sphere of influence, which has been used by some mission planners to estimate the region within which material could be stably trapped. We recall that the sphere of influence is defined as that surface along which it is equally valid to consider the motion of the particle relative to the Sun with the asteroid as a perturber as it is to consider the motion of the particle relative to the asteroid with the Sun as a perturber (Roy 1978). That is to say, the sphere of influence is the locus of points where the ratios of the perturbing forces to the direct forces in the two cases are equal. This sphere lies within the Hill sphere for $\mu<0.004$ but the difference only becomes significant (Chebotarev 1964) when $\mu$ is very small, as in the case under consideration here. Amphitrite's sphere of influence has a radius of $115 R_{\mathrm{A}}$.

As an example of scaling, we consider orbits about Galieleo's target asteroid 951 Gaspra, which has a semimajor axis of $2.2 \mathrm{AU}$. From its mean brightness, it is reasonable to assume a radius of $10 \mathrm{~km}$ for Gaspra, so that, if its density is taken to be $2.38 \mathrm{~g} / \mathrm{cm}^{3}$ (that chosen for Amphitrite), then $\mu=5 \times 10^{-15}$ for Gaspra. Our scaling law then states that to apply our Amphitrite plots given below to an asteroid with Gaspra's parameters, but zero orbital eccentricity, distances measured in asteroid radii should simply be reduced by the ratio of the semimajor axes, namely $2.55 / 2.2=1.16$.

\section{ANALYTIC ESCAPE CRITERIA}

Many estimates of analytical escape criteria for circular orbits have been made; most follow either from considering the Jacobi constant that will open the zero-velocity curves or from equating forces in a rotating frame (see Fig. 3). Szebehely (1978) has used the former method to predict that circular orbits will escape when they start beyond $r_{H} / 3$. Markellos and Roy (1981) refined Szebehely's treatment by including all of the terms in the Jacobi equation [Eq. (5) with $\mathrm{i}=0^{\circ}$ and $\mathrm{i}=180^{\circ}$ ] to derive critical distances of $\sim 0.49 r_{\mathrm{H}}$ for prograde circular orbits and $\sim 0.28 r_{\mathrm{H}}$ for retrograde circular orbits (see Fig. 4). These distances are lower limits for escape; particles starting on circular orbits within these distances are constrained by closed zero-velocity surfaces that encircle the asteroid. Our numerical results for initially circular orbits are $\sim 0.49 r_{\mathrm{H}}$ for prograde orbits and $\sim r_{\mathrm{H}}$ for retrograde ones (see Fig. 15). The agreement of the prograde results is impressive, while that of the retrograde results is appalling. But there is a simple explanation: the method outlined above ignores the influence of the Coriolis acceleration on the particle since the scalar product of the Coriolis term in Eq. (2) with $\mathbf{v}_{\text {rot }}$ is zero. The effect of this omission is abundantly clear in the results of Markellos and Roy which predict that retrograde orbits are less stable than prograde ones, even though the directions of prograde and retrograde Coriolis accelerations imply the converse (see Fig. 2). In fact, we find that prograde orbits slip away as soon as escape is energetically possible, pushed outward by the omitted Coriolis acceleration, while retrograde orbits linger, held in by this acceleration.

Equating forces in a rotating reference frame was originally applied by King (1962) who showed that direct gravity balances the "tidal" force along the $x$-axis at a distance $r_{\mathrm{H}}$. Innanen (1979) added the effects of the Coriolis force to obtain limiting radii for prograde and retrograde orbits of $0.69 r_{\mathrm{H}}$ and $1.44 r_{\mathrm{H}}$, respectively. This work contains a subtle error which involves the translation of the particle's velocity into the rotating frame; after correction of this mistake, we find that the limiting radii calculated via Innanen's method should be $0.80 r_{\mathrm{H}}$ and $2.60 r_{\mathrm{H}}$, respectively (these distances are the points where the normalized force curves $P$ and $R$ attain a value of zero in Fig. 3). This method shows that retrograde orbits are stable out to much greater distances than prograde ones, but gives poor agreement with numerical results (see the discussion of Fig. 3 for an explanation of why agreement is poor).

Various arguments (see, e.g., Keenan and Innanen 1975) have been given for the reason why retrograde orbits 


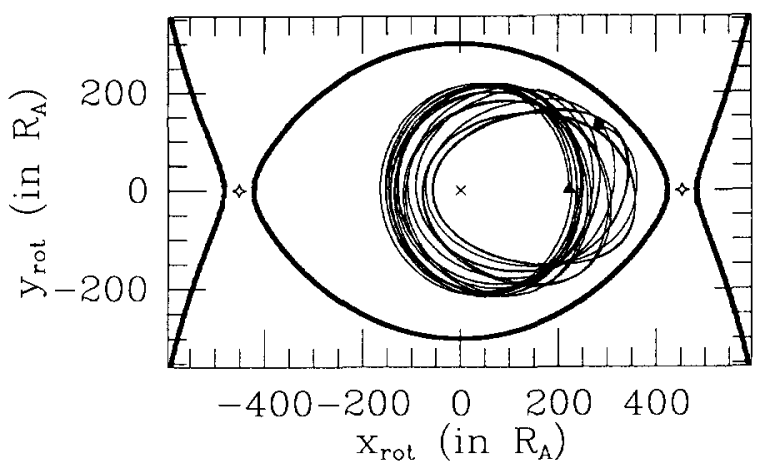

FIG. 5. The path of a particle started on a prograde coplanar circular orbit at $221 R_{\mathrm{A}}(C=9.0505)$ as seen in the rotating coordinate system. The asteroid's position is given by an $x$, the particle's initial location by the small triangle with one point showing the direction of the initial velocity, and the particle's location at the end of the integration by the solid square. The Sun lies out the negative $x_{\text {rot }}$-axis throughout the integration. The heavy line shows the zero-velocity curve specified by the initial conditions [see Eq. (4)] and the stars show the positions of the nearby Lagrange points (and accordingly the size of the Hill sphere).

are so much more stable than prograde ones, but one we find especially appealing relies on the nature of epicycles, the paths of particles on elliptical orbits as seen from a coordinate system that moves at the mean orbital rate; epicyclic motions are retrograde and, for small eccentricities, take place along a 2:1 ellipse aligned with the long axis in the direction of the orbital motion. That is to say, if a particle felt no gravitational attraction to the asteroid and had an elliptical path around the sun of the same semimajor axis as the asteroid's, it would be observed in the rotating system to travel along a retrograde path (see Chauvineau and Mignard 1990a). In a very real sense the retrograde motion is preferred whereas prograde motion must be forced.

\section{INDIVIDUAL EXAMPLES}

\section{Coplanar Trapped Orbits}

Our numerical experiments for the Amphitrite case show that all trajectories that start as circular prograde orbits within $\sim 224 R_{\mathrm{A}}(C=9.0000)$ are bound, while most of those outside this range escape from the asteroid. Since we are concerned with the outer limit where material can still be retained by the asteroid, we show an orbit (Fig. 5) that is close to the stability limit, namely one that was initially circular at $221 R_{\mathrm{A}}(C=9.0505)$. The displayed orbit is quasiperiodic with two dominant frequencies: one is the inverse of the synodic period, the other eight times slower as will be seen in Fig. 6. The regular appearance of this orbit in the rotating frame is due to the fact that the two dominant frequencies are close to a ratio of integers.
Relevant timescales are the asteroid's orbital period (4.08 Earth years), and the sidereal period of an unperturbed satellite at $221 R_{\mathrm{A}}(0.80$ years $)$. The unit of time in this and the following plots is taken to be an asteroid year (the period of the asteroid's orbit around the sun).

We can qualitatively understand the orbital evolution of Fig. 5 by considering the acceleration [Eq. (1)] along an initially circular orbit. At first, the path is elongated into an elliptical shape by the action of the tidal term since the Coriolis term does not change a circular orbit (an orbit that is circular in the sidereal frame will also be circular in the synodic frame; the Coriolis acceleration in this simple case merely accounts for the difference in orbital velocity measured in the two frames). As the orbit elongates and is flattened further, the Coriolis acceleration becomes increasingly asymmetrical (see Fig. 2); the strengthened Coriolis acceleration near the pericenter enhances radial accelerations there whereas the corresponding acceleration is diminished near apocenter (Fig. 3). In fact, the direction of the Coriolis acceleration near the apocenter can switch sign if the eccentricity is high enough [remember that it is the velocity in the rotating frame that appears in Eq. (1)]; although such a reversal does not occur in any of the planar orbits displayed in this paper, we have noticed it in some of our integrations. Now the
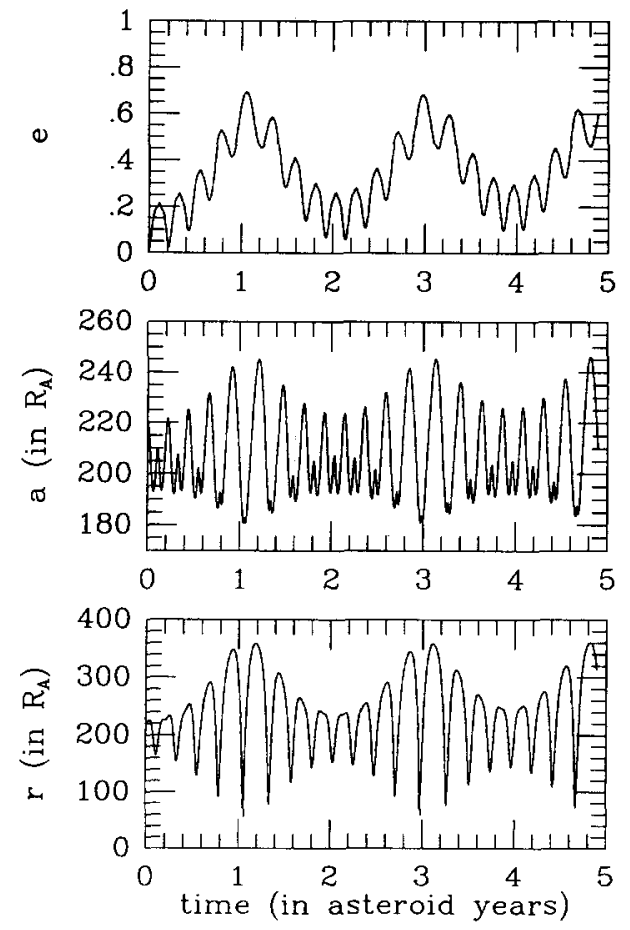

FIG. 6. The time history of the orbit shown in Fig. 5. Plotted are the osculating orbital eccentricity $e$, orbital semimajor axis $a$, and orbital radius $r$ as functions of time in asteroid years. The orbit is most perturbed when it is farthest from the asteroid. It is bound and almost periodic. 
fact that the Coriolis acceleration near the apocenter is less that that necessary to maintain a circular orbit allows local gravity to more effectively compete with the tidal force. This competition is most apparent in highly eccentric orbits where the apocenter end of the ellipse appears to be flattened (Fig. 5). The asymmetry of the Coriolis acceleration acts to circularize the orbit, and eventually it dominates the elongating effect of the tidal force. In the example under discussion, this occurs after the third synodic period. The elongation slows, stops, and reverses itself. The orbit then becomes more circular until the tidal force again dominates the Coriolis force and the process repeats. The period of this cycle is eight times the synodic period as was mentioned above.

The entire orbital path of the prograde satellite shown in Fig. 5 lies well within the zero-velocity curve, defined by Eq. (4), that corresponds to the initial conditions. This occurs because a significant fraction of the "energy" in the Jacobi integral remains in kinetic energy. It is apparent from the zero-velocity curve that the specified starting conditions have too little initial "energy" to allow escape.

The dynamical history of any particle can be described in terms of its initial vector position and velocity or, equally well, in terms of its four osculating orbital elements for a two-dimensional problem (Danby 1988). The osculating orbital elements are defined to be those that describe the conic section that the particle would follow if all perturbations were turned off. These elements, which we define in the nonrotating frame, change with time as perturbations cause the particle to deviate from true elliptical motion. Of the orbital elements, the orbital semimajor axis $a$ is the most significant when addressing escape since the size of the orbit, $2 a$, formally becomes infinite and then attains negative values as the particle goes through the escape process. The time histories of the osculating orbital elements that describe the path about the asteroid shown in Fig. 5 are displayed in Fig. 6. Here the periodic nature of the solution is clearly visible. We note that the semimajor axis vs. time curve has local extrema near the points where the orbit crosses the $x_{\text {rot }}$ and $y_{\text {rot }}$ axes. This feature arises because orbital energy is directly related to the semimajor axis (Burns 1976) and because the work done by the tidal force changes sign in each quadrant of the $x_{\text {rot }}-y_{\text {rot }}$ plane. In general, the work done by the tidal force will change sign four times in a single orbit, although this need not occur at the points where the orbit crosses the axes.

The histories of the other osculating orbital elements can be determined by solving the Lagrange perturbation equations and, although we have made a start on that study, we will not present it here.

In the next example (Fig. 7), the particle starts along a retrograde circular orbit twice as large as the first example; it begins at $x_{\text {rot }}=445 R_{\mathrm{A}}, y_{\text {rot }}=0(C=1.5518)$,

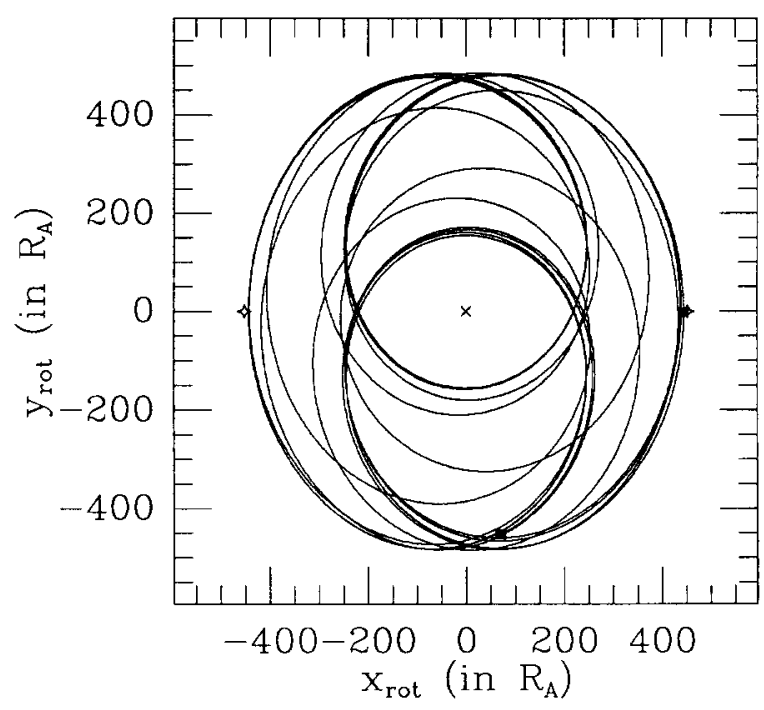

FIG. 7. The path of a particle started on a retrograde coplanar circular orbit at $445 R_{\mathrm{A}}(C=1.5518)$ as observed in the rotating coordinate system. See the legend to Fig. 5 for a description of the symbols. The orbit is bound and has a very regular appearance.

very close to the transition between bound and unbound retrograde orbits. The unperturbed sidereal orbital period is about 2.3 years or about $4 / 7$ of an asteroid year. As with prograde orbits, quasiperiodic retrograde orbits are also common; this one has two major frequencies that are not quite a ratio of integers (see Fig. 9). For this retrograde orbit the zero-velocity curves do not constrain the motion (Chauvineau and Mignard 1990a) since, as a result of the

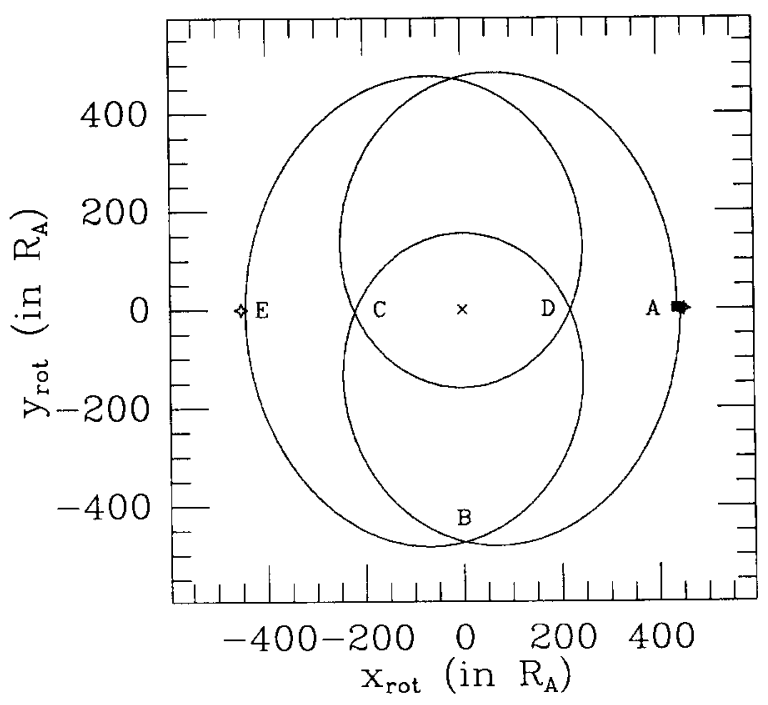

FIG. 8. The first few loops of the orbit shown in Fig. 7. The letters on the path are used in the text to describe various arcs along which particular accelerations dominate the motion. 

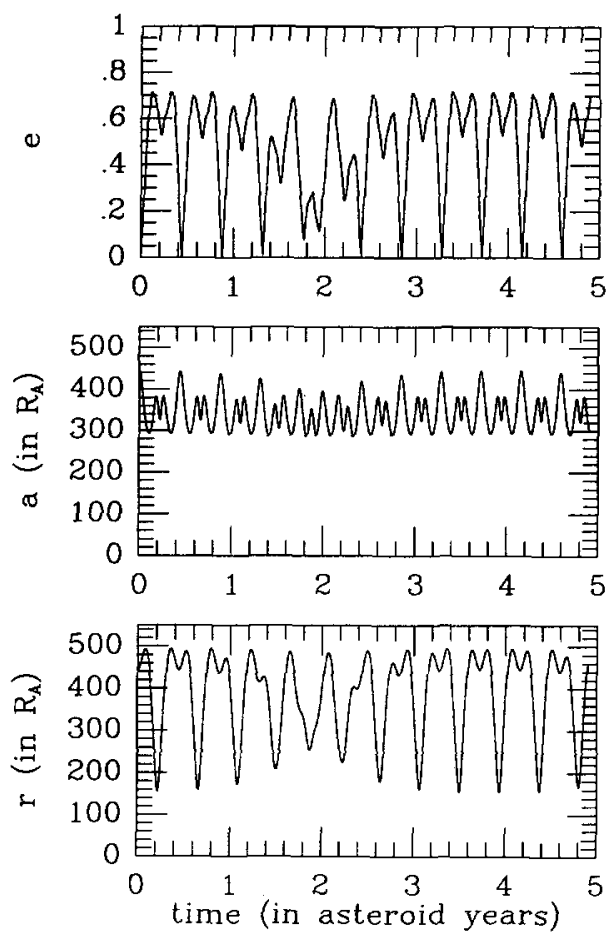

FIG. 9. The time histories of some osculating orbital elements for the retrograde orbit displayed in Fig. 7.

small $C$ (due to the large apparent velocity of a retrograde orbit as measured in the rotating frame), the curves do not enclose the asteroid. Nevertheless the particle is obviously bound; indeed we note that it is strongly influenced by the asteroid since its orbital shape is not the $2: 1$ ellipse characteristic of epicyclic motion.

To analyze the particle's motion, consider the perturbing effects of the tidal and Coriolis terms on a circular orbit (see Fig. 8, which shows the first three loops of Fig. 7). Initially the tidal term dominates, since the Coriolis acceleration does not change the shape of a circular orbit. This pushes the particle in the $x_{\text {rot }}$ direction ( $\operatorname{arc} \mathrm{AB}$ ) which displaces the orbit as a whole to the right (positive $x_{\text {rot }}$ ). When the particle moves to the left side of the asteroid, it is much closer to the asteroid due to this displacement (arc BC). Thus at point $\mathrm{C}$ the tidal term, being proportional to $x_{\text {rot }}$ (see Fig. 2), is smaller than it was at A. Hence the total contribution of the tidal force along $\mathrm{BC}$ is smaller than the integrated effect along $A B$, resulting in a net displacement of the orbit to the right. In addition, the Coriolis acceleration, which is stronger over arc $\mathrm{BC}$ than over arc AB due to a larger velocity, dominates the weakening tidal force. The particle then swings around the asteroid $(\operatorname{arc} \mathrm{CD})$, mostly under the influence of the asteroid's gravity, and out to large $r$ where Monsieur Coriolis starts to tug it to the left (arc DB). The tidal force switches sign again, and pulls the particle outward along arc BE to the point $\mathrm{E}$, where it has roughly the negative of its initial

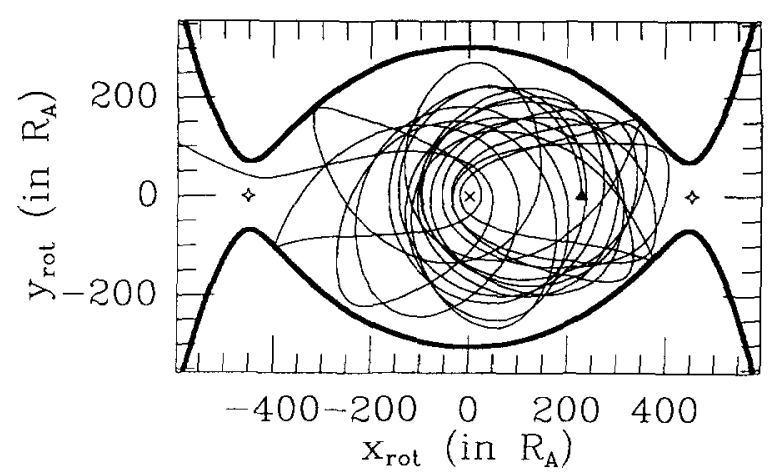

FIG. 10. The trajectory of a coplanar prograde particle that escapes after starting on a circular orbit at $227.25 R_{\mathrm{A}}(C=8.9423)$. The symbols are defined in the legend to Fig. 5. Note that, in contrast to Fig. 5 , the initial conditions here are such that the zero-velocity curve is open to heliocentric space and the particle, after bouncing chaotically around within the zero-velocity bottle, eventually slips out the neck to move along an elliptic heliocentric orbit having properties described in the text.

velocity and position: the cycle repeats. The histories of the osculating orbital elements $a$ and $e$ are presented in Fig. 9.

\section{Coplanar Escape Orbits}

Figures 10 and 11 show planar escape orbits that have initial conditions that are close to the bound orbits of Figs. 5 and 7 ; thus all of these orbits lie near the stability boundary. In those cases where escape is marginal (such

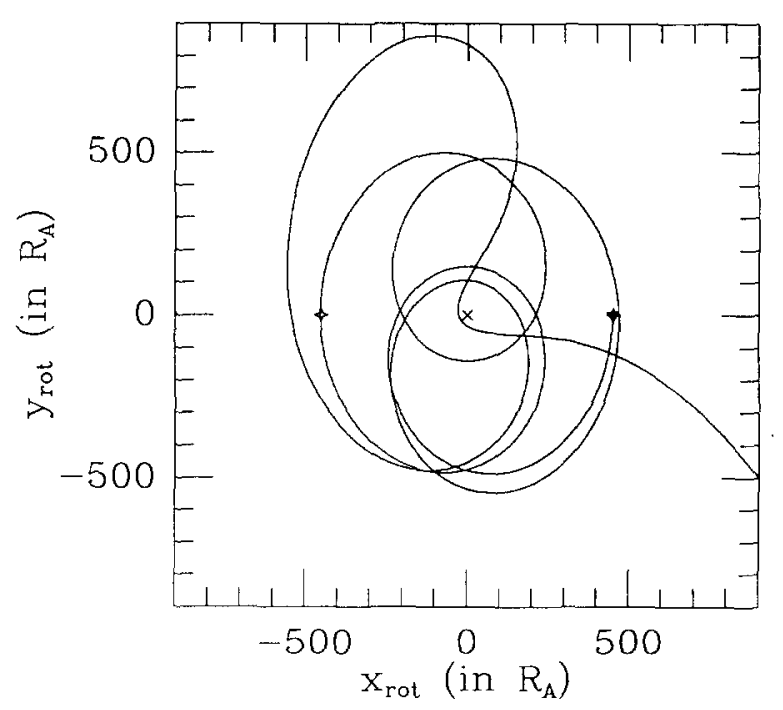

FIG. 11. The trajectory of a coplanar retrograde particle that escapes after starting on a circular orbit at $450 R_{\mathrm{A}}(C=1.5421)$. See the legend to Fig. 5 for a description of the symbols used. Note that on the last loop the path extends well beyond the radius of the Hill sphere and that the particle transfers to a prograde orbit before escaping. In this case, the transfer to a prograde orbit occurs in both the rotating and the nonrotating frames. The character of the escape path is discussed in the text. 
as all those discussed here), the direction of escape is always near the Sun-asteroid line because the outwardly directed tidal term is maximum there (Fig. 2). This result, which remains valid even for inclined orbits, can also be understood readily from the zero-velocity surface which opens first along the Sun-asteroid line (see Fig. 10). Of course, with large enough initial "energy" (or, equivalently, small enough $C$ for the zero-velocity curves to be wide open), objects can escape in any direction, but in all of the cases that concern us, objects depart from the asteroid with little extra energy because the particle is initially bound (i.e., its energy in the two-body system composed of the asteroid and the particle is initially negative) and the perturbation forces can modify this energy only slowly. In fact, the Coriolis acceleration, being perpendicular to the orbital velocity, can do no work and thus does not alter the orbital energy at all.

Figure 10 shows a chaotic prograde orbit started at $227.25 R_{\mathrm{A}}(C=8.9423)$ that escapes inward toward the Sun. The fact that the zero-velocity surface accurately delimits the accessible region of space is apparent. Note that since, by definition, speeds must be zero on zerovelocity surfaces, particles approach the surface perpendicular to it so as to form orbital cusps.

Because the asteroid's orbit is circular, one can very simply calculate the parameters of the solar orbit that is attained by escaping particles. Since the particle departs the asteroid with a very low velocity relative to the rotating frame, we can ignore this velocity as well as later influences of the asteroid (since it is so small and so distant) when estimating the particle's heliocentric energy which determines directly the orbital semimajor axis of the particle in its new path around the Sun (Burns 1976). The particle's velocity in the rotating frame is lowest near the inner Lagrange point (see Fig. 10), so at this point its angular velocity about the Sun closely matches that of the asteroid. Making the simplification that the particle starts from the inner Lagrange point with zero velocity in the rotating frame, one can calculate the specific (i.e., per unit mass) heliocentric kinetic energy of the particle $\Omega^{2}(R-$ $\left.r_{\mathrm{H}}\right)^{2} / 2$, and its specific potential energy $-G M /\left(R-r_{\mathrm{H}}\right)$. Equating the sum of these two energies to the total specific heliocentric energy in terms of semimajor axis $(E=$ $-G M / 2 a$ ), we find that the ratio of the semimajor axis $a$ of the particle's orbit about the Sun to the asteroid's semimajor axis $R$ is $a / R=\left(1-4 r_{\mathrm{H}} / R\right)$. Since the particle's initial velocity in the nonrotating frame is perpendicular to the solar direction and the particle initially falls toward the Sun, the Lagrange point must be at the aphelion of the new solar orbit. Solving the equation for aphelion $a(1+e)=R-r_{\mathrm{H}}$ yields (to first order) an eccentricity of $3 r_{\mathrm{H}} / R$. Since the escaped particle's semimajor axis is smaller than the asteroid's the particle's orbital period is shorter, so its path trails off to the upper left as viewed in the frame rotating with the asteroid's mean motion (Fig.

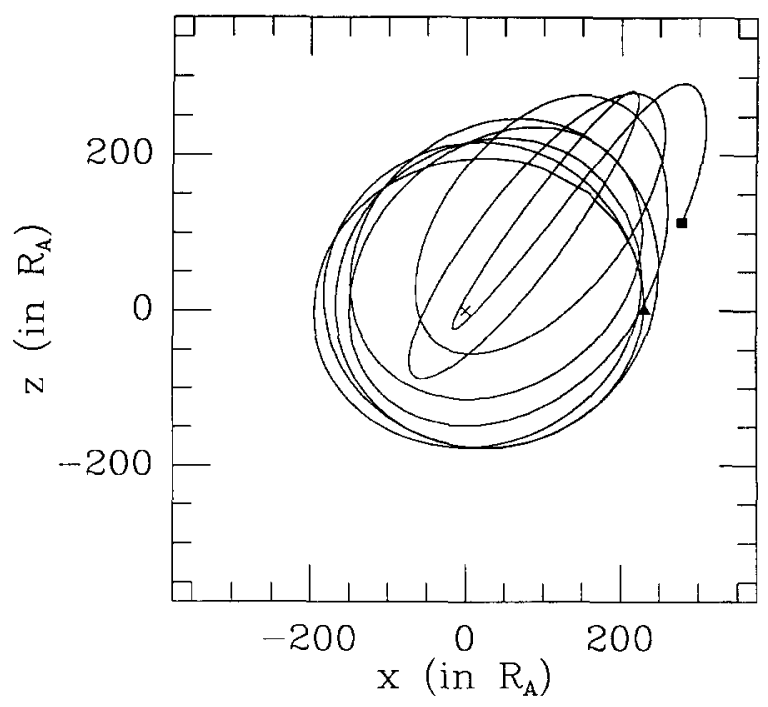

FIG. 12. The trajectory of a particle started on a circular orbit at 230 $R_{\mathrm{A}}$ with an inclination of $70^{\circ}$ as viewed in a projection onto the $x-z$ plane of the nonrotating system $(C=7.2747)$. The symbols are defined in the legend to Fig. 5. This particle eventually escapes.

10). Alternatively, the direction of departure can be understood in the rotating frame by considering the effects of the Coriolis acceleration.

Figure 11 shows a retrograde orbit starting at 450 $R_{\mathrm{A}}(C=1.5421)$ that becomes prograde just prior to escape. Arguments similar to those for the prograde orbit can be used to find $a^{\prime}=R+4 r_{\mathrm{H}}$ and $e^{\prime}=3 r_{\mathrm{H}} / R$; thus the

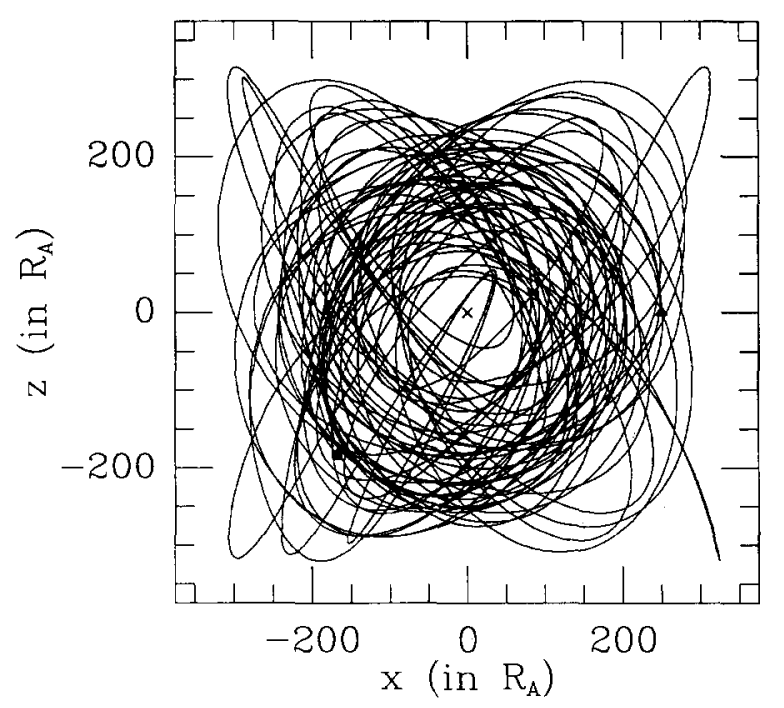

FIG. 13. An $x-z$ projection of a 50-year integration of a particle started on a circular orbit at $250 R_{\mathrm{A}}$ with an initial inclination of $70^{\circ}$ $(C=6.9306)$. This particle, like many others on three-dimensional orbits with inclinations satisfying $60^{\circ}<i<120^{\circ}$, is seen to reach roughly the same $z$ value regardless of $x$. 


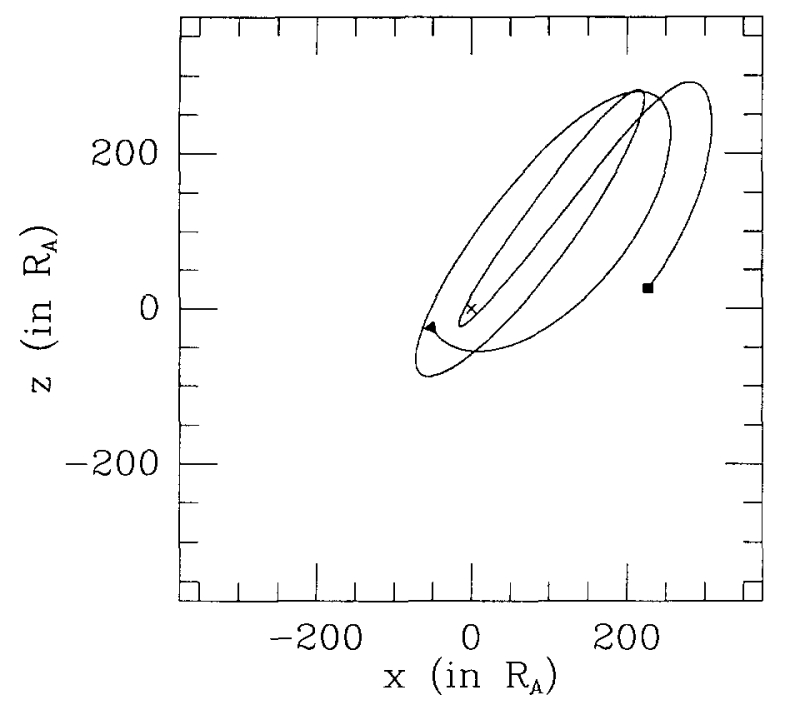

FIG. 14. Effect of tidal forces on an inclined elliptical orbit. Notice that the actual orbital path for a single revolution around the asteroid is displaced to the right from where an unperturbed elliptical path would lie. This, of course, is due to the tidal acceleration. The orbit shown is part of that in Fig. 12.

particle's escape path trails off to the lower right. Again, although it is not as clear as in the prograde case, the point of lowest relative velocity occurs near a Lagrange point.

\section{Inclined Orbits}

Figures 12 and 13, which are plotted in nonrotating coordinates, show orbits with initial inclinations of $70^{\circ}$. Figure 12, where the trajectory is seen as projected onto the $x-z$ plane, displays an orbit that starts out roughly circular at a distance of $230 R_{\mathrm{A}}(C=7.2747)$ but changes to an oval shape that becomes narrower and narrower until, on the last loop, the direction of rotation actually reverses! When viewed in three dimensions, the ellipse is tilted out of the asteroid's orbital plane by approximately $45^{\circ}$ and the direction of its major axis is such that the latter's projection onto the orbital plane lies along the initial Sun-asteroid line. The ellipse is not as narrow as it appears in this projection since it also extends in the $y$ direction. To lessen confusion in the diagram, we have elected not to show the further evolution of the orbit but will describe it. The highly eccentric orbit is seen to broaden slowly until it is approximately circular. At this point, the cycle begins to repeat with the circular orbit slowly becoming more eccentric, but after a second close approach to the asteroid, the particle escapes. In many orbits (e.g., Fig. 13) this cycle continues without an escape. Each time the approximately circular orbit begins to increase its eccentricity, the major axis of the new ellipse is found to be tilted at $\sim 45^{\circ}$ from the $x-y$ plane and to lie along the reoriented Sun-asteroid line. The axis can be tilted either toward or away from the Sun, and can lie either primarily above the $x-y$ plane or primarily below it due to the symmetry of the tidal term. Figure 13 shows an orbit started at $x=250 R_{\mathrm{A}}(C=6.9306)$ that was followed for 10 circuits of the asteroid around the Sun. Notice that the maximum $z$ values attained by the orbit are approximately independent of $x$. This characteristic, which was observed on many distant orbits with $60^{\circ}<i<120^{\circ}$, will have an important influence on the shape of the stability zone described below.

In the depicted case, tidal perturbations alone must be responsible for the motion since the results are plotted in nonrotating coordinates, where no Coriolis term appears. The form of the tidal perturbation in the nonrotating frame is $\Omega^{2}\left(3 x_{\text {rot }} \hat{\mathbf{x}}_{\text {rot }}-r_{\mathrm{i}}\right)$, which differs from the second term of Eq. (2) since that term included the centrifugal acceleration of the rotating frame. In the following, we lump the radial part of the tidal term in with the asteroid's gravity, and consider only the effects of the $x_{\text {rot }}$ term. Consider a particle that would be on an elliptical orbit primarily in the $x-z$ plane in the absence of perturbations (Fig. 14), and ignore for the moment the fact that the Sun is not always along the $x$-axis. We see that, starting from $x=0$, the tidal perturbation pushes the particle to larger values of $x$ than would be experienced in a two-body problem. Because of this added acceleration, the particle drops along an orbital path that brings it closer to the asteroid than its unperturbed counterpart. Throughout the region of close approach, the tidal force is negligible so that we can approximate the motion there by the solution to the two-body problem. Hence, after one revolution, the particle emerges on a more highly eccentric ellipse, and the cycle repeats. The outcome of the narrowing ellipse is either an impact with the asteroid or a reversal of the direction of rotation (see Fig. 14). If the latter occurs, the tidal acceleration operates in the opposite way to broaden the orbit out to a circle where the whole process begins anew. Because of passage through many of these very narrow ellipses, the probability for a particle on an orbit of this type to impact the asteroid is very large. We note that the reverse of such an impact orbit offers a mechanism by which material, blasted from the surface of the asteroid by a collision, could be put into distant orbits.

The essence of this argument is unchanged when we take into account that the Sun is not always along the $x$ direction as measured in the non-rotating frame. Therefore in general the tidal acceleration contains both $x$ and $y$ components that vary in time. Because the particle's orbital motion remains primarily in the $x-z$ plane, the direction of the tidal acceleration varies roughly sinusoidally as this plane moves with the asteroid's angular fre- 


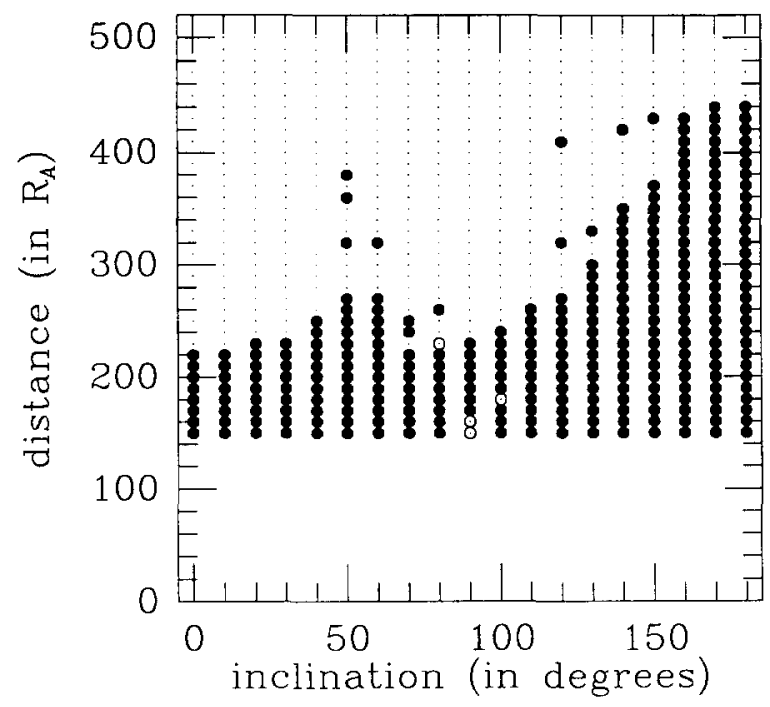

FIG. 15. The critical distance, which divides stable from unstable orbits, as a function of initial inclination. All particles are injected on initially unperturbed circular orbits along the Sun-asteroid line. A large solid dot signifies an orbit that remains near the asteroid for at least 5 asteroid years, a small dot is an orbit that escapes in less than this amount of time, and an open circle with a dot inside is an orbit that strikes the asteroid. Note that orbits with $i>90^{\circ}$, particularly those that approach purely retrograde orbits, are stable out to much greater distances than coplanar prograde paths (see text for discussion).

quency around the Sun. Thus generally the $x$-component of the tidal acceleration dominates the $y$-component for the simple reason that the orbit never samples large $y$ values. The argument can be generalized for orbits whose motions are primarily in the $x^{\prime}-z$ plane where $x^{\prime}$ is some linear combination of the $x$ and $y$ directions. Orbits with inclinations in the range $60^{\circ}<i<120^{\circ}$ have their motions primarily in some $x^{\prime}-z$ plane, and thus exhibit this type of dynamical motion.

\section{GLOBAL STRUCTURE}

\section{Escape as a Function of Inclination}

To explore the effects of orbital inclination on the stability of particles, we studied weakly bound orbits that began at various inclinations but otherwise chose the same initial conditions for purposes of comparison. We define the critical distance as the initial displacement within which most orbits remain bound, and outside of which most escape. We find that the critical distance displays a strong dependence on initial inclination. Naturally, because of the problem's fractal-like nature (Murison 1989b), occasional orbits within the critical distance escape, while some others outside this distance are bound; in this sense the critical surface is a very complex structure that cannot be truly represented by a single curve. The number of these exceptions, however, decreases rapidly as one moves away from the transition region.

Figure 15 shows the results of almost 700 different integrations in which the initial distance and initial inclination were varied in increments of $10 R_{\mathrm{A}}$ and $10^{\circ}$, respectively. The diagram distinguishes between orbits that escape, those that remain captured, and those that crash into the asteroid. Note that the collision orbits occur predominantly for inclinations around $90^{\circ}$ where orbits undergo the "narrowing ellipse" motion described above. It is apparent that there is a fairly crisp "boundary" between the bound and escape orbits; this boundary is the critical distance. Most of the graph's features can be interpreted as due to the Coriolis acceleration. Taking a circular orbit for illustration, consider the radial part of the Coriolis term (i.e., toward or away from the asteroid), which is proportional to $\cos i$ and which therefore attains its maxi-

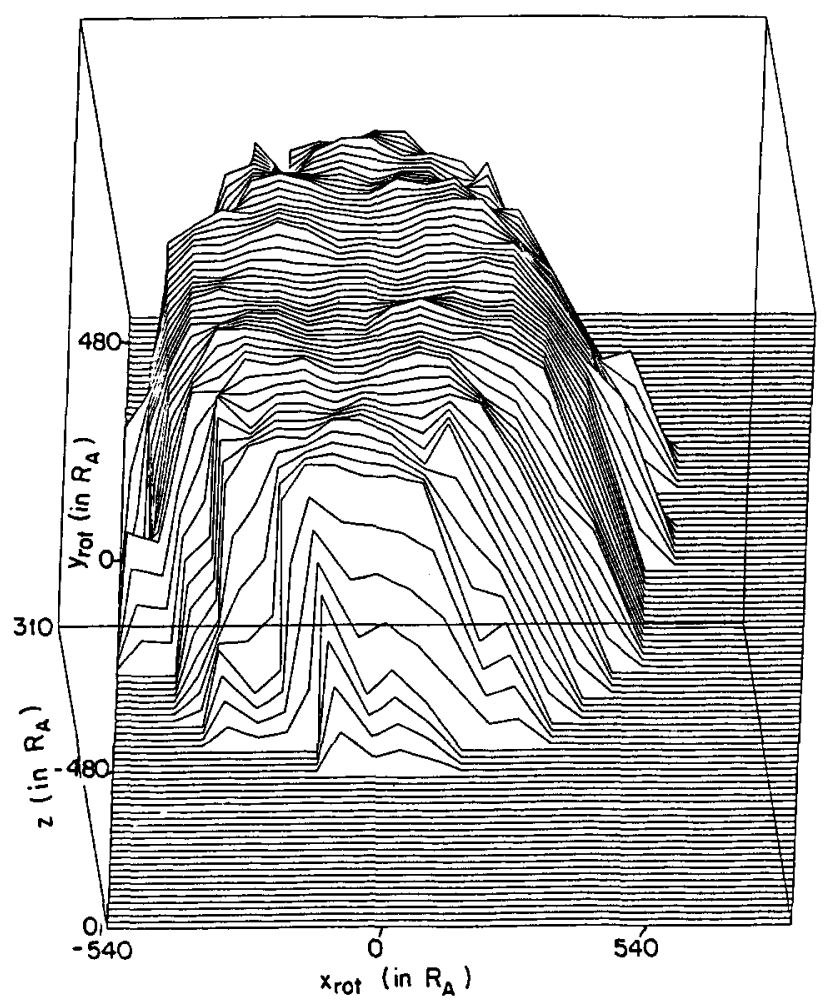

FIG. 16. Plot of the upper half of the stability surface viewed from pitch $=60^{\circ}$, yaw $=10^{\circ}$, and roll $=0^{\circ}$ as suggested by the reference cube. Note that the scale is distorted due to the viewing angle. The flattened top surface is at an approximate altitude of $z=285 R_{\mathrm{A}}$, and the surface drops off precipitously to the roughly circular base region $\left(r \sim 480 R_{A}\right)$. To determine this surface we took the exterior envelope of the orbits of about 1000 particles that were started near the critical distance but remained captured for 5 asteroid years. Thus, if pathological cases are ignored, particles found within the surface are generally bound to the asteroid while those outside are not. See the text's discussion for more details about how this figure was constructed. This figure clearly illustrates that stable orbits are more closely confined in the polar region. 
mum inward and outward strengths at $i=180^{\circ}$ and $i=0^{\circ}$, respectively. This predicts the upward trend of the critical distance with inclination in Fig. 15.

We find a local minimum in the critical distance near $i=90^{\circ}$, confirming previous results of Keenan (1981). This feature and the rough symmetry for $\pm 30^{\circ}$ around $i=$ $90^{\circ}$ can be explained by abrupt inclination shifts that we have observed in orbits with initial inclinations in the range $60^{\circ}<i<120^{\circ}$. We have found that many escape orbits with inclinations $i$ in this range switch to orbits with an inclination $\sim 180^{\circ}-i$ via the narrowing ellipse process outlined above (see the section on inclined examples), and thus escape for both $i$ and $180^{\circ}-i$ orbits can occur at the smaller inclination where the Coriolis binding acceleration is weaker. Together, these two effects predict the overall shape of Fig. 15. Nonradial Coriolis accelerations, which are maximum near $i=90^{\circ}$, may also influence the structure and exact location of the minimum.

\section{The "Stability Boundary"}

Figure 16 illustrates the shape of the boundary within which stable orbits lie. The surface represents the maximum $z$ value obtained by a particle as a function of $x_{\text {rot }}$ and $y_{\text {rot }}$, not for a single orbit, but for the union of the 239 stable orbits lying within the critical distance in Fig. 15. The rare stable orbits found in regions where unstable orbits predominate were not included (see prior discussion of the fractal-like nature of the stability boundary and Fig. 15). The output of our integration routine is a series of points in the rotating system $\left(x_{\mathrm{rot}}, y_{\mathrm{rot}}, z\right)$ through which a given orbit passes. We divided the $x_{\text {rot }}-y_{\text {rot }}$ plane up into a $20 \times 20$ grid of $60 \times 60-\mathrm{km}$ squares and recorded the maximum $z$ value occurring above each square from the union of all of the points in each of the stable orbits. The data were then interpolated out to an $80 \times 80$ grid to optimize the viewing.

We also exploited two symmetries to quadruple the effective number of input orbits to Fig. 16. It can be shown that the transformation of initial conditions $(z \rightarrow-z$, $v_{z} \rightarrow-v_{z}$ ) results in an orbit that is the reflection of the original orbit through the $x-y$ plane [see Eq. (2)]. This follows most simply from considerations of the symmetry of the gravitational forces in an inertial frame centered on the Sun. Thus each of our orbits has a mirror image through the $x_{\text {rot }}-y_{\text {rot }}$ plane and we can incorporate this image by taking not the maximum $z$, but the maximum $|z|$ attained. This effectively doubles the number of input orbits. Furthermore, the transformation $\left(\mathbf{r} \rightarrow-\mathbf{r}, \mathbf{v}_{\text {rot }} \rightarrow\right.$ $\mathbf{v}_{\text {rot }}$ ) also yields identically shaped orbits in Hill's problem, so we can again double the number of input orbits. All told, there are almost 1000 separate initial conditions incorporated in Fig. 16, each pertaining to an orbit that is stable for at least 5 asteroid years.
Figure 16 shows that the stability surface is roughly flat on top with very steep sides. The plateau region is at an average height of about $285 R_{\mathrm{A}}$ above the $x-y$ plane with the highest orbit rising to $307 R_{\mathrm{A}}$ above the plane; its base is roughly circular with a radius of about $480 R_{\mathrm{A}}$. The flattened polar region arises from the fact that maximum $z$ values attained by orbits with $60^{\circ}<i<120^{\circ}$ are roughly independent of $x$ and $y$ (see Fig. 13). The plotted surface is not based on enough different orbits to validate comments on its second order structure; in addition, we remind the reader that this surface pertains to particular initial conditions, and thus the detailed shape may change somewhat with different modes of injection.

\section{DISCUSSION}

We have found that the stability zone consisting of the vast majority of bound orbits resembles a circular mesa with precipitous edges rising to a height of $z=$ $285 R_{\mathrm{A}}$. Due to the effect of the tidal acceleration on orbits with $60^{\circ}<i<120^{\circ}$, none of our orbital trajectories rise above $z=310 R_{\mathrm{A}}$ and only a few rise above $z=$ $300 R_{\mathrm{A}}$. Beyond this surface (i.e., more than $310 R_{\mathrm{A}}$ above or below the asteroid's orbital plane), no stable orbiting particles can exist unless trapped on a pathologic path. Furthermore, many orbits that populate large $z$ values on this diagram would in fact be lost since they have a high probability of colliding with the asteroid as previously noted. As we have seen, particles on stable prograde coplanar orbits can be found out to $360 R_{\mathrm{A}}$ (see, e.g., Fig. 6) but material on stable coplanar retrograde orbits reaches nearly $500 R_{\mathrm{A}}$ (Fig. 9). Thus, if debris on prograde and retrograde orbits are equally likely, these results argue in favor of having the spacecraft's close approach (especially for the flyby of the first one!) take place out of the orbital plane.

The stability surface shown in Fig. 16 corresponds to the case where the asteroid is on a circular orbit and the only perturbations on the system are due to the gravitational attraction of the Sun. Many effects that we have ignored here and will take up in a later paper will reduce the size of the stability zone. Radiation pressure, for example, will sweep centimeter and smaller-sized particles from the system in less than one orbit of the asteroid around the Sun. The remarkable efficiency of these solar photons is due to the fact that the gravitational attraction of the asteroid is really quite small. Perturbations from the planets, especially Jupiter, cause a drift in the value of the Jacobi constant, thereby allowing some previously bound particles to escape (Whipple and White 1985, Chauvineau and Mignard 1990b), as will asteroids that pass close to-or even through-the system.

Finally, one would expect an asteroid with a nonzero 
orbital eccentricity to have a smaller zone of stability than one with zero eccentricity for several reasons. First, eccentricity will cause the asteroid to periodically have a closer approach to the Sun, at which point the Hill sphere is smaller, and hence escape occurs more readily. Second, in the elliptic problem the equation of motion will contain an additional acceleration term that is destabilizing for most orbits. We have run several simulations for asteroids with nonzero orbital eccentricity and these numerical results, as well as the results of Zhang and Innanen (1988), verify that the zone of stability is reduced; this result will be presented elsewhere.

The boundary discussed above is only a limit on where bound orbits can exist, not an absolute boundary within which debris must be found. Since it depends not only on which orbits are stable but also on how material is supplied to the region, the location of any actual debris cloud, it is likely to be much closer to the asteroid. In fact, it is not known whether circum-asteroidal debris will be prevalent enough to threaten a spacecraft at all. Clearly mechanisms for populating orbits must be included in any estimate of the location of material orbiting an asteroid, and therefore in any spacecraft trajectory planning. Most schemes (Weidenschilling et al. 1989) to populate stable debris orbits favor paths of much smaller size than those considered here, suggesting that a safe distance of close approach could be considerably smaller than the distances that we have computed. Future evaluations of the debris hazard about asteroids should be focused on bettering our understanding of population mechanisms for circumasteroidal particles. Only once these mechanisms are understood can one fully answer the question of how close an asteroid flyby should be.

\section{ACKNOWLEDGMENTS}

We thank P. J. Holmes and P. D. Nicholson for helpful discussions and critical readings of the text. We appreciate the prompt and careful reviews received from A. Dobrovolskis, A. Harris, and F. Mignard. Barbara $\mathbf{L}$. Boettcher provided valuable assistance in preparing the figures. We were encouraged to complete this work by various members of the Galileo mission, including T. V. Johnson, J. Veverka, and the late C. Yeates.

\section{REFERENCES}

BurNs, J. A. 1976. An elementary derivation of the perturbation equations of celestial mechanics. Amer. J. Phys. 44, 944-949 (Erratum: Amer. J. Phys. 45, 1230).
Chauvineau, B., And F. Mignard 1990a. Dynamics of binary asteroids. I. Hill's case. Icarus 83, 360-381.

Chauvineau, B., AND F. Mignard 1990b. Dynamics of binary asteroids. II. Jovian perturbations. Icarus 87, 377-390.

Chebotarev, G. A. 1964. Gravitational spheres of the major planets, Moon and Sun. Soviet Astron.-AJ 7, 618-622.

DANBY, J. M. A. 1988. Fundamentals of Celestial Mechanics, 2nd ed. Willmann-Bell, Richmond, VA.

Gradie, J., H. Hammel, and C. Prlcher 1985. A search for material around asteroid 29 Amphitrite. Bull. Amer. Astron. Soc. 17, 729.

HÉNON, M. 1970. Numerical exploration of the restricted problem. VI. Hill's case: Non-periodic orbits. Astron. Astrophys. 9, 24-36.

Hénon, M., AND J. M. Petit 1986. Series expansions for encountertype solutions of Hill's problem. Cel. Mech. 38, 67-100.

INNANEN, K. A. 1979. The limiting radii of direct and retrograde satellite orbits, with applications to the solar system and to stellar systems. Astron. J. 84, 960-963.

Keenan, D. W. 1981. Galactic tidal limits on star clusters. II. Tidal radius and outer dynamical structure. Astron. Astrophys. 95, 340-348.

KeEnan, D. W. and K. A. InNanen 1975. Numerical investigation of galactic tidal effects on spherical stellar systems. Astron. J. 80, 290-302.

KING, I. 1962. The structure of star clusters. I. An empirical density law. Astron. J. 67, 471-485.

MARKellos, V. V., AND A. E. RoY 1981. Hill stability of satellite orbits. Cel. Mech. 23, 269-275.

Murison, M. A. 1989a. On an efficient and accurate method to integrate restricted three-body orbits. Astron. J. 97, 1496-1509.

Murison, M. A. $1989 \mathrm{~b}$. The fractal dynamics of satellite capture in the circular restricted three-body problem. Astron. J. 98, 23462359.

Press, W. H., B. P. Flannery, S. A. Teukolsky, and W. T. VetterING 1987. Numerical Recipes, the Art of Scientific Computing, pp. 547-577. Cambridge University Press, Cambridge.

Roy, A. E. 1978. Orbital Motion, 2nd ed. Adam Hilger, Bristol.

SzeBeHely, V. 1967. Theory of Orbits: The Restricted Problem of Three Bodies, Academic Press, New York/London.

Szebehely, V. 1978. Stability of artificial and natural satellites. Cel. Mech. 18, 383-389.

Terrile, R. J., AND B. A. Smith 1985. The coronagraphic search for material around Amphitrite. Bull. Amer. Astron. Soc. 17, 918.

Weidenschilling S. J., P. PaOlicchi, and V. ZapPala 1989. In Asteroids II (R. P. Binzel, T. Gehrels, and M. S. Matthews, Eds.), pp. 643-658. Univ. Ariz. Press, Tucson.

WhIPPLE, A. L., AND L. K. WHITE 1985 . Stability of binary asteroids. Cel. Mech. 35, 95-104.

WisDom, J. 1982. The origin of the Kirkwood gaps: A mapping for asteroidal motion near the $3 / 1$ commensurability. Astron. J. 87, 577-593.

ZhaNG, S. P., AND K. A. INNANEN 1988. The stable region of satellites of large asteroids. Icarus 75, 105-112. 